Vol. 69, N. ${ }^{\circ}$ IIO (noviembre), 25-37

\title{
¿QUIÉN DEBE PAGAR IMPUESTOS ? UN ANÁLISIS DE MICROSIMULACIÓN PARA EL IMPUESTO A LA RENTA DE PERSONAS NATURALES EN EL ECUADOR
}

\author{
MAURICIO CUESTA ${ }^{1}$, XAVIER JARA ${ }^{2}$ \\ ${ }^{1}$ Instituto de Altos Estudios Nacionales, Ecuador; \\ ${ }^{2}$ Institute for Social and Economic Research, University of Essex, UK
}

Recepción de manuscrito: 11 de septiembre de 2017

Aceptación del manuscrito: 23 de octubre de 2017

\begin{abstract}
RESUMEN El presente estudio utiliza ECUAMOD, el modelo de microsimulación de impuestos y transferencias sociales del Ecuador, para caracterizar al individuo típico que debe pagar impuesto a la renta en base a la Encuesta Nacional de Ingresos y Gastos de los Hogares Urbanos y Rurales 2011-2012. Nuestros resultados ${ }^{1}$ muestran que el aporte de la mayoría de los contribuyentes representa $0,6 \%$ de la recaudación total, mientras que los pocos contribuyentes que ganan más contribuyen con el $27,5 \%$ de la recaudación total. El ingreso laboral es el mayor determinante de la probabilidad de pagar impuesto a la renta, pero se observan diferencias en cuanto a otros determinantes entre hombres y mujeres. Nuestro estudio concluye con una discusión de las posibilidades que ofrece la microsimulación para el análisis de reformas tributarias en el futuro.
\end{abstract}

PALABRAS CLAVE Impuestos, microsimulación, Ecuador, probit.

ABSTRACT This study uses ECUAMOD, the tax-benefit microsimulation model for Ecuador, to characterize the typical individual who must pay income tax based on information from the National Survey on Income and Expenditure of Urban and Rural Households 2011-2012. Our results show that the contribution of the majority of taxpayers represents $0,6 \%$ of total tax revenue, while the few taxpayers who earn the most contribute with $27,5 \%$ of tax revenue. Labor income is the major determinant of the probability of paying income tax but differences are observed in terms of other determinants between men and women. Our study concludes with a discussion of the possibilities offered by microsimulation for future analysis of tax reforms.

KEYWORDS Taxes, microsimulation, Ecuador, probit

JEL CODES $\mathrm{D}_{3} \mathrm{O}, \mathrm{H}_{53}, \mathrm{I}_{3} 8$

\section{INTRODUCCIÓN}

Los impuestos son transferencias obligatorias de recursos de individuos a instituciones públicas con el propósito de proveer recursos materiales con los cuales financiar las obras que han sido asignadas a las instituciones públicas (Menéndez, 2001, p. 20). La imposición de impuestos ${ }^{2}$ a los ingresos de los individuos es tan antigua como la humanidad, se cobraba incluso en especie 
— como el rey Minos en Creta - productos, trabajo, seres humanos. En la Biblia se habla de la obligación de contribuir con el diezmo para Dios y los sacerdotes -Deuteronomio-. En Roma se grababa impuestos a las colonias conquistadas — centésima-, impuestos de importaciónexportación - portoria - a la explotación de tierras públicas - ager publicus- (Menéndez, 2001, p. 90). En Grecia se creaba un impuesto para financiar las guerras - eisfora-. En los tiempos feudales todos sus súbditos estaban obligados a contribuir con los resultados de su trabajo - talla - al señor feudal. En 1799, Inglaterra establece el impuesto del 10\% a los ingresos para financiar las guerras napoleónicas — precursor del impuesto moderno-. En 1913, en Estados Unidos, el impuesto al ingreso se establece como la principal fuente de ingresos para el gobierno (Gómez et al., 2010, pp. 10-16).

En el Ecuador, los conceptos básicos tributarios surgen en la colonia para financiar el estado colonial: el quinto real a la explotación minera. A mediados del siglo XVII la minería pierde protagonismo y se crean otros impuestos: almojarifazgo, sobre bienes importados; sisa, rebaja en pesas a favor del Estado; alcabala, impuesto sobre todo tipo de transacción comercial; lanzas, pago anual a poseedores de título de nobleza; diezmo, pago a la iglesia; estancos, estatización de ciertos productos; impuesto a los indios, a ingresos especiales - mesada, media annata, servicios especiales, pensión del censo, donativos graciosos- (Paz y Miño, 2015, pp. 59-62).

Los impuestos no es un asunto técnico, sino político, probablemente el más polémico de todos los asuntos políticos. Se trata de ponerse de acuerdo sobre quién debe pagar qué y en nombre de qué principios (Piketty, 2014, p. 493); responden a la visión política y los intereses sociales que determinan las acciones de un gobierno (Paz y Miño, 2015, p. 230).

La cuestión de la desigualdad y la redistribución está en el centro del conflicto político. La posición liberal dice que solo las fuerzas de mercado, la iniciativa individual y el crecimiento de la productividad permiten mejorar los ingresos y las condiciones de vida, y que, por lo tanto, la acción pública debe limitarse a herramientas que interfieran lo menos posible con ese mecanismo virtuoso. La posición de izquierda dice que solo las luchas sociales y políticas pueden aliviar la indigencia de los más necesitados producida por el sistema capitalista, y que la política pública de redistribución debe cuestionar la manera en que las fuerzas del mercado determinan las ganancias del capital así como las desigualdades entre asalariados (Piketty, 2015, p. 9).

Los debates acerca de la reforma de política para el desarrollo, en general, se centran alrededor de la mejora de los servicios sociales, en la efectividad de los programas de reducción de la pobreza, el rol del comercio y la liberalización del mercado, pero muy raramente se ocupan de reformas tributarias y la necesidad de desarrollar sistemas modernos de recaudación de impuestos (Piketty and Quian, 2010, p. 40).

Por ejemplo, el reporte sobre el desarrollo mundial del Banco Mundial, desde su inicio, 1998, se ocupa de temas sobre conocimiento, pobreza, mercado, servicios, inversión, igualdad, agricultura, geografía, clima, seguridad, empleo, riesgo, gobernanza y educación para el desarrollo (World Bank, 2017); ningún análisis o tratamiento de los impuestos como factor de financiamiento o apoyo al desarrollo de los países.

En el Ecuador, los impuestos ${ }^{3}$ representan la principal fuente de ingresos para financiar el presupuesto del gobierno. En el 2016, del total de ingresos, que fue de USD 29.835 millones, los impuestos representaron el 51,9\% del presupuesto; considerando solo ingresos permanentes, 
en el 2011, los impuestos representaron el 83,9\% (Ministerio de Economía y Finanzas, 2016). Por otro lado, de la programación presupuestaria proyectada para el 2015 -USD 19.407 millones-, el ingreso tributario representaba el 69\% de los ingresos totales del gobierno; y el 29,2\% de la recaudación tributaria proviene del pago del impuesto a la renta de los ciudadanos y empresas (Ministerio de Economía y Finanzas, 2012).

El presente estudio describe e identifica al individuo típico que debe pagar impuesto a la renta en el Ecuador usando técnicas de microsimulación de impuestos y transferencias monetarias en base a información detallada de encuestas de hogares. En particular, el análisis hace uso de ECUAMOD, el modelo de microsimulación de impuestos y transferencias para el Ecuador que utiliza información disponible en la Encuesta Nacional de Ingresos y Gastos de los Hogares Urbanos y Rurales (ENIGHUR) 2011-2012 la cual permite obtener resultados representativos a nivel nacional (Instituto Ecuatoriano de Estadística y Censos, 2012). La estimación de pago de impuestos por medio de microsimulación consiste en aplicar las reglas de cálculo de impuesto a la renta de personas naturales a cada uno de los individuos en la encuesta a partir de sus ingresos laborales y gastos deducibles reportados en la ENIGHUR.

La microsimulación ha sido usada crecientemente como herramienta para determinar el impacto distribucional ex ante de reformas de política tributaria y de transferencias sociales. En el caso de países de Europa, el modelo de microsimulación EuROMOD se ha convertido en un referente para el análisis distribucional comparativo de reformas fiscales y de transferencias sociales, y ha sido utilizado en numerosos estudios. Figari, Paulus y Sutherland (2015) muestran una revisión del uso de modelos de microsimulación para evaluar el impacto de políticas públicas. Recientemente modelos de microsimulación para países en desarrollo han sido construidos en base a EUROMOD con el objetivo de proveer herramientas para el análisis de reformas redistributivas (Instituto Mundial para el Desarrollo e Investigación Económica de la Universidad de la Naciones Unidas, 2017).

Técnicas de microsimulación han sido aplicadas anteriormente para el caso del Ecuador aunque aún con poca frecuencia. Rossignolo, Oliva y Villacreses (2016) llevan a cabo un análisis a partir de microsimulaciones que resultan de aplicar la estructura del impuesto a la renta sobre los datos de la encuesta Encuesta Nacional de Empleo y Desempleo Urbano (ENEMDU) para los períodos 2011-2014. Las estimaciones muestran la existencia de un gravamen sobre los ingresos personales con una elevada progresividad, y donde el $20 \%$ de la población con más altos ingresos paga más del 90\% del impuesto, pero muy bajo impacto redistributivo (Rossignolo, Oliva y Villacreses, 2016, p. 37).

También, Cano (2016) utiliza datos del pago de impuesto a la renta compilado por la administración de impuestos ecuatoriana: el Servicio de Rentas Internas (SRI), mediante microsimulación, calcula aritméticamente el efecto de una reducción $-50 \%-$ y eliminación $-100 \%-$ de deducciones del impuesto a la renta en la distribución del ingreso para cada contribuyente. Su estudio concluye que, aunque el pago del impuesto en Ecuador es altamente progresivo, su capacidad de redistribución del ingreso es baja (Cano, 2016).

Nuestro análisis sigue esta misma dirección y utiliza el modelo ECUAMOD para caracterizar al individuo típico que debe pagar impuesto a la renta en el Ecuador. Los resultados de las simulaciones de ECUAMOD han sido previamente validados con respecto a estadísticas 
administrativas (Jara et al., 2017). Recientemente, ECUAMOD también ha sido utilizado para analizar el impacto redistributivo de políticas fiscales y de transferencias sociales en el Ecuador (Jara y Varela, 2017).

El resto del documento está organizado como sigue. En la segunda parte se describe brevemente el sistema de impuesto a la renta vigente en el Ecuador en el 2011. En la sección 3 se describe sucintamente el modelo de microsimulación ECUAMOD y se compara los resultados de nuestras simulaciones de impuesto a la renta de personas naturales, en particular la estimación de la distribución del número de contribuyentes por tramos de pago de impuesto utilizando ECUAMOD, con la distribución reportada por Chiliquinga y Villacreses (2017) en base a datos administrativos del SRI. En la sección 4 se describen las características de los pagadores de impuesto a la renta: demografía, familia, educación, dónde se ubica geográficamente, dónde y en qué trabaja, condiciones de seguro y cuál es el promedio de pago de impuestos, para finalmente en la sección 5 poder definir las características del contribuyente típico con su respectiva probabilidad de ocurrencia. La última sección termina con una discusión de nuestros resultados y posibles extensiones al modelo ECUAMOD.

\section{EL IMPUESTO A LA RENTA EN EL ECUADOR}

La recaudación de impuestos en el 2011 provino de cinco impuestos y otros ingresos relacionados con la recaudación como son las multas e intereses y otros. Dentro de este esquema de política fiscal, el impuesto a la renta representa un tercio de los ingresos del Estado (ver Tabla 1).

Del 2000 al 2007, el impuesto a la renta se pagaba en base a ingresos distribuidos en seis franjas de ingresos básicos con un mínimo de ingreso que no paga impuestos y las cinco restantes pagaban una tasa de impuesto incremental de $5 \%$ hasta $25 \%$. Durante todo el período se mantuvo la misma imposición de pago. A partir del 2008, el pago del impuesto a la renta se modifica a nueve franjas de ingresos con un mínimo de ingreso que no paga impuesto - franja 1 - y las ocho restantes tienen un incremento variable desde 5\% - franja 2- sobre excedentes hasta un pago máximo de 35\% - franja 9- (ver Tabla 2) (Servicio de Rentas Interno, 2017).

La imposición progresiva es probablemente una herramienta de política menos distorsionadora para mantener bajo control el aumento de la desigualdad y para redistribuir un poco más equitativamente las ganancias del crecimiento. Es menos distorsionador que otros instrumentos más radicales como la nacionalización, los salarios mínimos o la autarquía (Piketty and Quian, 2010, p. 41).

\section{ECUAMOD Y LOS DATOS}

El modelo ECUAMod es parte del proyecto southmod desarrollado por el Instituto Mundial para el Desarrollo e Investigación Económica de la Universidad de la Naciones Unidas (UNUWIDER), el grupo EUROMOD ${ }^{4}$ en el Instituto de Investigación Económica y Social (ISER) de la Universidad de Essex y el Instituto de Investigación de Política Social Sudafricano (sAsPRI), en el cual se han diseñado modelos de microsimulación para Ecuador, Etiopia, Ghana, Mozambique, Namibia, Sudáfrica, Tanzania, Vietnam y Zambia. 
Tabla 1. Recaudación de impuestos en 2011

\begin{tabular}{lcc}
\hline Impuestos & Millones de uSD & Porcentaje \\
\hline Valor agregado & 4,958 & $52 \%$ \\
Renta & 3,112 & $33 \%$ \\
Consumos especiales & 618 & $7 \%$ \\
Salida de divisas & 491 & $5 \%$ \\
Vehículos motorizados & 174 & $1,8 \%$ \\
Intereses y multas tributarias & 59 & $0,6 \%$ \\
Otros ingresos & 50 & $0,5 \%$ \\
TOTAL & 9,462 & $100 \%$ \\
\hline
\end{tabular}

Fuente: SRI (2017).

Tabla 2. Tasa marginal y monto mínimo a pagar por impuesto a la renta de personas naturales (2011)

\begin{tabular}{lccccccccc}
\hline \multicolumn{1}{c}{ Franja } & $\mathbf{1}$ & $\mathbf{2}$ & $\mathbf{3}$ & $\mathbf{4}$ & $\mathbf{5}$ & 6 & 7 & 8 & 9 \\
\hline Tasa sobre el excedente & 0 & $5 \%$ & $10 \%$ & $12 \%$ & $15 \%$ & $20 \%$ & $25 \%$ & $30 \%$ & $35 \%$ \\
Fracción básica (USD) & 9,21 & 11,73 & 14,67 & 17,61 & 35,21 & 52,81 & 70,42 & 93,89 & $>93,890$ \\
Impuesto mínimo (USD) & 0 & 0 & 126 & 420 & 773 & 3413 & 6933 & 11.335 & 18.376
\end{tabular}

Fuente: SRI (2017).

ECUAMOD combina reglas detalladas de política tributaria y de transferencias sociales con microdatos de la Encuesta Nacional de Ingresos y Gastos de Hogares Urbanos y Rurales (ENIGHUR) para simular el pago del impuesto a la renta de personas naturales y contribuciones al seguro social, así como transferencias del Bono de Desarrollo Humano (BDH) y el bono Joaquín Gallegos Lara. Actualmente, ECUAMOD cubre los años 2011 al 2016 para simulaciones de impuestos y transferencias monetarias. ${ }^{5}$ La principal ventaja de usar ECUAMOD es su capacidad para estimar los efectos de cambios en impuestos y beneficios en la distribución del ingreso y simular potenciales reformas de política tomando en cuenta las complejas interacciones entre políticas de impuesto y transferencias, al igual que la heterogeneidad de la población.

Nuestro análisis utiliza ECUAMOD, el modelo de microsimulación de impuestos y transferencia para el Ecuador basado en datos representativos de hogares, la encuesta ENIGHUR 20112012, la cual contiene datos detallados de ingreso y gastos así como características de hogares y de individuos. El tamaño de la muestra es de 153.341 individuos pertenecientes a 39.617 hogares. Para este estudio se utilizan simulaciones de políticas vigentes en el año 2011 - año de base de la encuesta - para la caracterización de los contribuyentes del impuesto a la renta de personas naturales. Adicionalmente, ECUAMOD permite simular impuestos y transferencias para los años 2012 al 2016 en base a información de la ENIGHUR 2011-2012. En estos casos, ingresos laborales y otros ingresos no simulados en el modelo son ajustados por medio de deflactores para tomar en cuenta la evolución de estos ingresos en el tiempo.

Las simulaciones de impuesto a la renta de personas naturales en ECUAMOD se realizan bajo el supuesto de cero evasiones. Como veremos a continuación, a pesar de este supuesto, nuestras simulaciones capturan relativamente bien el número total y la distribución de contribuyentes 
del impuesto a la renta, al igual que el monto de la recaudación. La gran ventaja de utilizar información de encuestas en conjunto con técnicas de microsimulación es que se permite caracterizar en detalle a los contribuyentes de impuestos o receptores de bonos, debido a la información detallada de características personales y de hogares disponibles en encuestas.

Antes de describir las características de los contribuyentes al impuesto a la renta, y con el objetivo de validar los resultados de nuestras simulaciones, presentamos una comparación del número de contribuyentes y recaudación de impuesto a la renta, obtenidos con ECUAMOD con respecto a estadísticas del SRI para los años 2011-2015 (ver Tabla 3).

El número de contribuyentes y la recaudación de impuesto a la renta son levemente subestimados en nuestras simulaciones con respecto a datos del SRI. Las simulaciones capturan mejor el número de contribuyentes y recaudación en el 2015. Es muy probable que la subestimación de nuestras simulaciones esté relacionada a la dificultad de capturar el tope de la distribución de ingresos en la ENIGHUR. Éste es un problema que afecta en general a las encuestas de hogares y de forma particular a encuestas de países en desarrollo.

A parte del número total de contribuyentes, es importante comparar su distribución por tramos de impuesto a la renta. La tabla (ver Tabla 4) presenta la distribución de contribuyentes por tramos de impuesto a la renta obtenidos con las simulaciones de ECUAMOD y los calculados por el SRI para el 2015. Se presenta igualmente el porcentaje de recaudación por tramo de ingreso obtenido por medio de las simulaciones de ECUAMOD.

En general, la distribución del número de contribuyentes obtenida con ECUAMOD refleja bien la distribución según datos del SRI: el comportamiento simulado por ECUAMOD con el reportado por el SRI para 2015 sigue el mismo patrón: la mayor proporción de contribuyentes pagan menos de USD 100. Alrededor de 27,1\% de los contribuyentes paga menos de USD 100 de acuerdo a nuestras simulaciones, mientras el porcentaje respectivo es de 30,5\% según el SRI. Es importante resaltar que nuestras simulaciones no capturan a ningún individuo en el tramo de impuesto a la renta superior a USD 1.000.00o, lo que sugiere la dificultad de la encuesta en capturar personas en el tope de la distribución de ingresos.

Sin embargo de la alta concentración de contribuyentes en el tramo de pago del impuesto de menos de USD $100-30,5 \%$, según el SRI-, vemos que éstos contribuyen solamente con el 0,6\% de la recaudación total, mientras que los que pagan entre USD 100.000 y USD 1.000.000, que representan el o,2\% de los contribuyentes, pagan el 27,5\% de la recaudación total. Esto nos indica que los que más ganan más pagan de impuestos, lo que muestra que existe una redistribución del ingreso.

En efecto, si se compara el coeficiente de Gini de ingreso de mercado con el coeficiente de Gini de ingreso disponible, se observa una reducción de 0,502 a 0,460 en el 2011. ${ }^{6}$ La contribución de impuestos a la reducción del Gini es de 0,009 puntos, es decir, casi un punto porcentual, lo que demuestra que la política fiscal en lo que se refiere al cobro de impuesto a la renta y su redistribución de beneficios, alcanza una distribución del ingresos un poco más igualitaria - a menor valor del coeficiente de Gini mayor igualdad en la distribución del ingreso-. Esto, considerando los contribuyentes que se capturan en la ENHIGUR, ya que como reportan Rossignolo, Oliva y Villacreses (2016), la desigualdad es mayor que la que resulta de las mediciones convencionales realizadas con las encuestas cuando se consideran los 
Tabla 3. Número de contribuyentes y recaudación del impuesto a la renta simulado por ECUAMOD y calculado por el SRI (2011-2015)

\begin{tabular}{lcccccc}
\hline & \multicolumn{2}{c}{$\begin{array}{c}\text { Número de contribuyentes } \\
\text { (en miles) }\end{array}$} & \multicolumn{3}{c}{$\begin{array}{c}\text { Recaudación } \\
\text { (millones de dólares) }\end{array}$} \\
\cline { 2 - 6 } & ECUAMOD & SRI & RATIO & ECUAMOD & SRI & RATIO \\
\hline 2011 & 331 & 472 & 0,7 & 615 & 784 & 0,78 \\
2012 & 368 & 494 & 0,75 & 711 & 856 & 0,83 \\
2013 & 403 & 503 & 0,8 & 804 & 935 & 0,86 \\
2014 & 444 & 510 & 0,87 & 896 & 1022 & 0,88 \\
2015 & 448 & 492 & 0,91 & 933 & 1019 & 0,92 \\
\hline
\end{tabular}

Fuente: Jara et al. (2017).

Tabla 4. Distribución del número de contribuyentes por tramos de pago del impuesto a la renta simulado por ECUAMOD y calculado por el SRI (2011-2015)

\begin{tabular}{rcccc}
\hline $\begin{array}{r}\text { Pago impuesto a } \\
\text { la renta (USD) }\end{array}$ & ECUAMOD & ECUAMOD & SRI ${ }^{(\text {a) }}$ & ECUAMOD \\
\cline { 2 - 5 } & $\begin{array}{r}\text { Número de } \\
\text { contribuyentes }\end{array}$ & $\begin{array}{c}\text { Porcentaje de } \\
\text { contribuyentes }\end{array}$ & $\begin{array}{c}\text { Porcentaje de } \\
\text { contribuyentes }\end{array}$ & $\begin{array}{c}\text { \% de la } \\
\text { recaudación total }\end{array}$ \\
$100-200$ & 121,126 & 27,1 & 30,5 & 0,6 \\
$200-500$ & 79,122 & 14,3 & 13,8 & 0,9 \\
$500-1000$ & 61,562 & 17,7 & 16,7 & 2,8 \\
$1000-2000$ & 49,825 & 13,8 & 12,4 & 4,8 \\
$2000-5000$ & 47,238 & 11,1 & 10,0 & 15,9 \\
$5000-10.000$ & 13,547 & 10,6 & 10,1 & 9,9 \\
$10.000-50.000$ & 8,782 & 3,0 & 3,5 & 18,5 \\
$50.000-100.000$ & 1,635 & 2,0 & 2,6 & 11,4 \\
$100.000-1.000 .000$ & 1,009 & 0,4 & 0,31 & 27,5 \\
$>1.000 .000$ & - & 0,2 & 0,17 & - \\
TOTAL & 447,69 & - & 0,01 & 100 \\
\hline
\end{tabular}

Nota: (a) la columna de SRI es de autoría de Chiliquinga y Villacreses (2017). Fuente: elaboración propia.

ingresos concentrados por los tramos más afluentes con información de registros impositivos (Rossignolo, Oliva y Villacreses, 2016).

\section{¿QUIÉN ES EL CONTRIBUYENTE?}

De acuerdo a nuestras simulaciones, cuando se expande la muestra para el análisis y se obtiene representatividad a nivel nacional en el 2011, tenemos que existen 1.327.588 personas que pagan impuestos. De estas personas, 331.450 pagan impuesto a la renta. A continuación describimos 
las características principales de los contribuyentes al impuesto a la renta de personas naturales. Para determinar el perfil del contribuyente se usa la moda de la distribución de las variables descriptivas como se reportan en la encuesta de hogares. Adicionalmente, en la siguiente sección se estima un modelo econométrico - probit - para determinar qué características influyen más en la probabilidad de pago del contribuyente.

De todos los contribuyentes al impuesto a la renta, $40 \%$ son mujeres y el $60 \%$ hombres. Loja es la provincia que tiene el mayor porcentaje de contribuyentes mujeres: $53,8 \%$ ante $46,2 \%$ hombres; mientras que Orellana es la de menor proporción de mujeres: $25 \%$ ante $75 \%$ hombres. El $62,1 \%$ es casado, el $27,3 \%$ soltero y $5 \%$ divorciado. Étnicamente, los mestizos son el $86,5 \%$, blancos el 6,3\% y los indígenas $2,1 \%$, el restante $5,2 \%$ de los contribuyentes se reparten en otro origen étnico como mulato, negro, afroecuatoriano o montubio.

En cuanto a la localización, el 90\% se encuentra en el sector urbano y $10 \%$ en el rural. Pichincha es la provincia que concentra el mayor porcentaje de contribuyentes con $33,1 \%$, seguida por Guayas con 24,4\%, Azuay 5,42\% y Manabí 5,17\%; estas cuatro provincias representan el $68 \%$ de las personas que pagan impuesto a la renta; el $32 \%$ restante se distribuye en las otras 19 provincias.

Según la experiencia laboral ${ }^{7}$ del contribuyente, la moda de la experiencia está entre los contribuyentes con menos de 10 años de trabajo, 57,3\%, seguido por los de 11 a 20 años, 21,9\%, y de los de 21 a 30 años, $15,2 \%$.

Por educación, los contribuyentes están repartidos aproximadamente en cinco categorías de educación: no educación $1,2 \%$, primaria $6 \%$, secundaria 19,8\% — hasta seis años de educación secundaria, $6,13 \%$ y hasta seis años $13,7 \%$ - postsecundaria, $2 \%$, y terciaria $71 \%$. Las provincias donde el número de mujeres contribuyentes con educación terciaria es superior al de hombres son Santo Domingo: 63,3\%, Imbabura: 59,4\%, Morona Santiago: 59,4\% y Los Ríos: $58 \%$. En general, se podría afirmar que las provincias que tienen contribuyentes mejor educados — globalmente provincial— son Loja, Guayas, Pichincha, Pastaza, en ese orden.

La mayor parte de los contribuyentes están afiliados al seguro general del Instituto Ecuatoriano de Seguridad Social (IESS): 68,4\%. El 9,7\% pertenece a otro tipo de seguro: seguro social voluntario: $3,8 \%$, seguro social campesino: $0,8 \%$, seguro social militar o de la policía: 5,12\% y seguro municipal: 0,01\%. El 21,9\% restante no cuenta con ningún tipo de seguro, de éstos, el 28,3\% está en Pichincha, 22,6\% en Guayas, 6,5\% en Manabí y 5,9\% en Azuay. Es decir, más de dos tercios, $63,3 \%$, de los contribuyentes que no cuentan con seguro se concentran en estas cuatro provincias.

El 19\% de los contribuyentes trabaja en comercio, 16,2\% en administración pública, 15,6\% en manufactura, y 11,4\% en educación; juntos, representan casi dos tercios de los contribuyentes $(62,1 \%)$, el restante $37,9 \%$ trabaja en transporte y comunicación, salud, bienes raíces, agricultura, finanzas, turismo, construcción, y otras actividades, en ese orden de importancia por el número de contribuyentes que se desempeñan en cada industria. Además, el 34,2\% de los contribuyentes se ocupa como profesional, el 15,5\% como vendedor, $14,3 \%$ son gerentes, el 10,9\% técnicos.

Por otro lado, el $28,7 \%$ de los profesionales trabaja en educación, el 61,4\% de los vendedores trabajan en comercio, 25,3\% de los gerentes trabaja en manufactura. Las industrias que albergan mayoría de mujeres son educación $73,4 \%$, salud $66,9 \%$, finanzas $66,9 \%$ y turismo $59 \%$. 
Tabla 5. Recaudación, número de contribuyentes y pago promedio de impuesto a la renta por contribuyente por provincia

\begin{tabular}{lcccccc}
\hline \multicolumn{2}{c}{ Recaudación (USD) } & \multicolumn{2}{c}{$\begin{array}{c}\text { Número de } \\
\text { contribuyentes }\end{array}$} & \multicolumn{2}{c}{$\begin{array}{c}\text { Pago de impuesto } \\
\text { promedio (USD) }\end{array}$} \\
\hline Pichincha & 250.766 .479 & $(1)$ & 109,784 & $(1)$ & 2,284 & $(3)$ \\
Guayas & 180.089 .622 & $(2)$ & 80,866 & $(2)$ & 2,227 & $(4)$ \\
Santo Domingo & 29.254 .862 & $(3)$ & 6,92 & $(12)$ & 4,228 & $(1)$ \\
Tungurahua & 20.014 .146 & $(4)$ & 11,566 & $(5)$ & 1,73 & $(6)$ \\
Azuay & 18.379 .006 & $(5)$ & 17,968 & $(3)$ & 1,023 & $(13)$ \\
Manabí & 13.526 .854 & $(7)$ & 17,146 & $(4)$ & 789 & $(16)$ \\
Orellana & 9.611 .166 & $(11)$ & 3,749 & $(15)$ & 2,564 & $(2)$ \\
\hline
\end{tabular}

Nota: números en paréntesis son el orden provincial. Fuente: elaboración propia.

Mientras que los hombres en su mayoría se dedican a la construcción: $86,3 \%$, agricultura: $82,9 \%$, transporte: $81,7 \%$ y manufactura: $79 \%$. Las mujeres se ocupan como profesionales: $25,6 \%$, gerentes: $17,5 \%$, vendedoras: $15 \%$; los hombres, por otro lado, mayoritariamente se desempeñan como profesionales: $46,9 \%$, vendedores: $16,3 \%$, técnicos: $14,1 \%$.

El contribuyente de Santo Domingo, en promedio, paga más impuesto a la renta, es decir, tiene mayores ingresos a nivel nacional, el pago promedio de impuestos es USD 4228 al año, seguido por el de Orellana, USD 2564 y Pichincha con USD 2284. Según el número de contribuyentes por provincia, Pichincha, Guayas y Azuay son las provincias con mayor número de contribuyentes, Santo Domingo está en la posición 12 en número de contribuyentes. Combinando el promedio de pago con el número de contribuyentes, las provincias de mayor recaudación son Pichincha, Guayas, Santo Domingo, Tungurahua y Azuay, en ese orden (ver Tabla 5).

\section{EL CONTRIBUYENTE TÍPICO}

El contribuyente típico es hombre: 60\%, de edad mediana 43 años, étnicamente mestizo: $86,5 \%$, casado: $62,1 \%$, con educación terciaria: $71 \%$. Se localiza en el sector urbano $-90 \%-$ de Pichincha o Guayas - 57,5\%-. Trabaja en el sector de comercio, administración pública, o manufactura: 50,8\% y se desempeña como profesional, vendedor o gerente: $64 \%$, afiliado al seguro general del IEss: $68,4 \%$, con una experiencia laboral menor a 10 años: $57,3 \%$, y paga USD 2260 de impuesto a la renta al año — promedio de Guayas y Pichincha-.

Ahora, es importante determinar qué características influyen más en la probabilidad de pagar impuesto a la renta. Para esto, utilizamos un modelo de regresión probit. El modelo es estimado para hombres y mujeres por separado con el objetivo de analizar si los determinantes de la probabilidad de pagar impuestos varían de acuerdo al género de la persona. Nuestra muestra de análisis está formada por las personas que tienen ingreso laboral positivo y la variable dependiente toma los valores 1 si la persona paga impuesto a la renta, o o de otra manera.

Los principales resultados muestran que, como es de esperar, de todas las características consideradas, la que mayormente aporta a la probabilidad de que una persona pague impuestos 
son los ingresos laborales, que duplican el valor de la pseudo $\mathrm{R}^{2}$. En el caso de los hombres, la pseudo $\mathrm{R}^{2}$ pasa de 0,2969 a 0,7089 cuando se incluyen ingresos laborales en la estimación. En el caso de las mujeres, la pseudo $\mathrm{R}^{2}$ aumenta de 0,3930 a 0,7258.

$\mathrm{Si}$ asumimos que una mayor probabilidad de pago de impuestos se debe a mayores ingresos y que éstos se deben a mayor edad, experiencia - años de trabajo - y educación, vemos que los resultados confirman la teoría económica del «capital humano» (Mincer, 1958, 1962; Shultz, 1960, 1061; Becker, 1962, 1964), donde a mayor edad la probabilidad de pago de impuestos crece a tasa decreciente para hombres y mujeres; también, a mayor educación mayor probabilidad, aunque los coeficientes son significativos solo para los hombres; y en ambos casos los años de trabajo presentan un coeficiente positivo aunque no significativo.

El origen étnico no es determinante en poder establecer si la persona tiene mayor o menor probabilidad de pagar impuestos. Por el contrario, su estado civil sí es determinante, y el efecto varía entre hombres y mujeres. Para los hombres, estar casado disminuye la probabilidad de pagar impuesto a la renta, mientras estar separado o divorciado la aumenta. Para las mujeres, vemos el efecto contrario: estar casada aumenta la probabilidad de que pague impuesto, mientras la probabilidad es menor si la persona es divorciada, separada o viuda.

En general, la industria en la que interviene no es determinante en la probabilidad de pagar impuesto. Para los hombres, trabajar en la administración pública o en el sector de educación disminuye su probabilidad de pagar impuesto a la renta. Para las mujeres, la probabilidad aumenta en el sector de transporte y finanzas. Con respecto a la ocupación, la probabilidad es mayor para los profesionales, oficinistas y maquinistas, en el caso de los hombres. Para las mujeres, la ocupación no es determinante de la probabilidad excepto que ésta disminuye para las artesanas.

Finalmente, solo algunas provincias son determinantes de la probabilidad de pagar impuesto y se observan diferencias entre hombres y mujeres. En ambos casos, la probabilidad disminuye para personas que viven en la provincia de Zamora Chinchipe. Para los hombres, la probabilidad también disminuye para la provincia del Cañar y en el caso de las mujeres para la provincia de Chimborazo. La provincia de Galápagos presenta un coeficiente positivo aunque significativo solo para las mujeres.

\section{CONCLUSIONES}

El presente estudio ha utilizado el modelo de microsimulación de impuestos y transferencias sociales para el Ecuador, ECUAMOD. El modelo permite aplicar las reglas de cálculo del impuesto a la renta, aportes al seguro social, bono de desarrollo humano y bono Joaquín Gallegos Lara, a cada una de las personas de la encuesta ENIGHUR 2011-2012 con el objetivo de analizar el impacto distribucional de instrumentos de política pública.

Nuestro análisis consistió en utilizar ECUAMOD para aplicar las reglas de cálculo de impuesto a la renta de personas naturales del año 2011 a los individuos de la encuesta y determinar las características del contribuyente típico. En resumen, la mayor parte de los contribuyentes son pequeños contribuyentes - menos de USD $100=27,1 \%$ - para obtener una recaudación del o,6\% del total, mientras que los pocos contribuyentes que ganan mucho - más de USD 100.000 
$=0,2 \%$ ) contribuyen con el $27,5 \%$ de la recaudación total; lo que nos indica que la política tributaria cumple con el objetivo de redistribuir la riqueza.

Los resultados son útiles para establecer en detalle las características de los contribuyentes en ese año con el objetivo de simular reformas al impuesto a la renta a futuro. Desde un punto de vista normativo, es importante conocer las características de las personas que deben pagar impuestos. Por ejemplo, es importante analizar si hay diferencias a nivel de género o etnicidad para entender más acerca de las razones que explican por qué ciertos grupos tengan mayor probabilidad de pagar impuestos. Nuestro estudio representa el primer paso hacia este tipo de análisis y muestra diferencias en los determinantes de la probabilidad de pagar impuesto a la renta entre hombres y mujeres.

Desde el punto de vista geográfico, nuestro análisis es sugestivo para la discusión de políticas tributarias regionales. Si el número de contribuyentes es mayor en ciertas provincias, se podría analizar, por ejemplo, el impacto de descentralizar el impuesto a la renta y aumentar la progresividad en ciertas provincias.

Nuestros resultados muestran algunas direcciones para extender el modelo ECUAMOD. Por ejemplo, nuestras simulaciones subestiman el impuesto a la renta de personas naturales con respecto a la información administrativa del SRI, lo cual está relacionado con la dificultad de capturar el tope de la distribución de ingresos en la ENIGHUR. La imputación de ingresos al tope de la distribución en la encuesta, representaría una extensión importante para el modelo. Igualmente, existe la necesidad de incorporar de alguna manera la evasión de impuestos dentro de los cálculos.

Futuros estudios deberían explotar la posibilidad de analizar reformas al sistema de impuesto a la renta con ECUAMOD. Se podría analizar, por ejemplo, el efecto de reformas a las deducciones legales, las cuales reducen el ingreso sobre el cual se calcula el pago de impuestos utilizado por individuos de altos ingresos cuya tasa de impuestos puede ser tan baja como el $7 \%$ para el $1 \%$ de ingresos más alto (Cano, 2016). Además, se debe explorar la tasa de impuesto óptima para hacer la recaudación de impuestos más redistributiva y las opciones de política para su aplicación.

\section{NOTAS}

1 Los resultados presentados aquí se basan en ECUAMOD v1.o. El modelo ECUAMOD es desarrollado, mantenido y administrado por Instituto Mundial para el Desarrollo e Investigación Económica de la Universidad de la Naciones Unidas (UNU-WIDER, por sus siglas en inglés) en colaboración con el equipo de EUROMOD del Instituto de Investigación Económica y Social (ISER) de la Universidad de Essex, con el Instituto de Investigación de Política Social Sudafricano (SASPRI) y socios locales de países en desarrollo seleccionados -Etiopía, Ghana, Mozambique, Zambia, Ecuador y Vietnam - en el ámbito del proyecto southmoD. El socio local de ECUAmod es el Instituto de Altos Estudios Nacionales (IAEN). Estamos en deuda con muchas personas que han contribuido al desarrollo de SOUTHMOD y ECUAMOD. Los resultados y su interpretación presentados en esta publicación son de exclusiva responsabilidad de los autores.

2 Los impuestos son una institución política. Son una imaginación institucional humana. Aparentemente no es apropiado hablar de ciertas contribuciones como impuestos antes de la modernidad (Menendez, 2001, p. 325). 
3 Los impuestos que incluye la recaudación tributaria son: impuesto a la renta, impuesto al valor agregado (IVA), impuesto a los vehículos, impuesto a consumos especiales (ICE), impuesto a la salida de divisas, aranceles y otros.

4 Para más información acerca de EUROMOD, ver Sutherland and Figari (2013).

5 Para mayor información sobre ECUAMOD, ver Jara et al., 2017.

6 ECUAMOD calcula el ingreso disponible como el ingreso de mercado más transferencias privadas y beneficios sociales menos impuestos y menos contribuciones al seguro social.

7 Definimos experiencia laboral como el número de años de trabajo reportado por los sujetos en la encuesta.

\section{REFERENCIAS}

Becker, G. (1962). Investment in human capital: A theoretical analysis. Journal of Political Economy, 72(3), 428-440.

Becker, G. (1964). Human capital: a theoretical and empirical analysis, with special reference to education. The Economic Journal, 76(303), 635-638.

Cano, L. (2016, junio). Personal income tax and income inequality: Ecuador 2007-2011. ResearchGate. Recuperado de https://www.researchgate.net/publication /311910010_Personal_Income_Tax_ and_Income_Inequality_Ecuador_2007-2011

Chiliquinga, D. y N. Villacreses (2017, enero). ¿Quiénes pagan impuestos en el Ecuador? Centro de Estudios Fiscales. Servicio de Rentas Interno. Recuperado de https://cef.sri.gob.ec/pluginfile. php/32891/mod_page/content/85/NR_1701.pdf.

Cuesta, M. y Jara, H. X. (2017, July). Poverty and vulnerability to poverty in Ecuador: a microsimulation approach. Paper presented at the Public Economics for Development. Maputo, Mozambique.

Figari, F., Paulus, A. and Sutherland, H. (2015). Microsimulation and policy analysis. Handbook of Income Distribution, (2B), 2141-2221.

Gómez, G., Amézquita, A., Hernández, J., Ramírez, M., Cortés, J., Delgado, E. y Márquez, M. (2010). Tratamiento fiscal de las asociaciones en participación. Eumed. Recuperado de https://www. eumed.net/libros/2011a/912/

Instituto Ecuatoriano de Estadística y Censos (INEC). 2012. Encuesta Nacional de Ingresos y Gastos de los hogares urbanos y rurales 2011-2012. Resumen metodológico y principales resultados. INEC. Recuperado de http://www.inec.gob.ec/Enighur_/Analisis_ENIGHUR\%202011-2012_rev.pdf

Jara, H. X., and Varela, M. (2017, octubre). Tax-benefit microsimulation and income redistribution in Ecuador. UnU-wider. Recuperado de https://www.wider.unu.edu/sites/default/files/Publications/ Working-paper/PDF/wp2017-177.pdf

Jara, H. X., Varela, M., Cuesta, M. and Amores, C. (2017). southmod Country report Ecuador. ECuAmoD v1.o. 2011-2016. Helsinki, Finland: UNU-WIDER.

Menéndez, A. J. (2001). Justifying taxes. Some elements for a general theory of democratic tax law. Springer Science. Recuperado de https://scholar.google.com/citations?user=x-vb3sIAAAAJ\&hl=es

Mincer, J. (1958). Investment in human capital and personal income distribution. Journal of Political Economy, 66(4), 281-302.

Mincer, J. (1974). Schooling, experience and earnings. New York, USA. University Press for the National Bureau of Economic Research.

Ministerio de Economía (2012). Programación presupuestaria cuatrienal 2012-2015. Ministerio de Economía y Finanzas. Recuperado de http://www.finanzas.gob.ec/indicadores-fiscales/ 
Ministerio de Economía y Finanzas (MEF) (2016). Estadísticas fiscales. Ministerio de Economía y Finanzas. Recuperado de http://www.finanzas.gob.ec/estadisticas-fiscales/

Paz y Miño, J. J. (2015). Historia de los impuestos en el Ecuador. Quito, Ecuador: SRI-PUCE-THE.

Piketty, T. (2014). Capital in the twenty first century. Cambridge, USA: Harvard University Press.

Piketty, T. (2015). La economía de las desigualdades. Cómo implementar una redistribución justa y eficaz de la riqueza. Buenos Aires, Argentina: Siglo xxi Editores.

Piketty, T. and Quian, N. (2010). Income Inequality and Progressive Income Taxation in China and India, 1986-2015. En A.B. Atkinson and Thomas Piketty (Eds.), Top incomes. A global perspective. Oxford, UK: Oxford University Press.

Rossignolo, D., Oliva, N. y Villacreses, N. (2016). Cálculo de la concentración de los altos ingresos sobre la base de los datos impositivos. Un análisis para el Ecuador. Macroeconomía del Desarrollo. Cepal. Recuperado de https://repositorio.cepal.org/handle/11362/40925

Servicio de Rentas Internas (SRI) (2017). Tarifas. Servicio de Rentas Internas. Recuperado de http://www. sri.gob.ec/de/167

Shultz, T. (1961). Investment in Human Capital. American Economic Review, 51(1), 1-17.

Shultz, T. (1960). Capital formation by education. Journal of Political Economy, 68(6), 571-583.

Sutherland, H. and Figari, F. (2013). Euromod: the European Union tax-benefit microsimulation model. International Journal of Microsimulation, 6(1), 4-26.

United Nations University World Institute for Development Economics Research (UNU-WIDER) (2017). SOUTHмоD. Simulating tax and benefit policies for development. UNU-WIDER. Recuperado de https://www.wider.unu.edu/project/southmod-simulating-tax-and-benefit-policies-development

World Bank. (2017). World Development Report. World Bank. Recuperado de http://www.worldbank. org/en/publication/wdr/wdr-archive 
